\section{CHEMICAL ACCIDENTS}

\section{0}

\section{Poison Center (PC) Participation in a Municipal Chemical Disaster Exercise}

Vicas IMO

Poison and Drug Information Service

Foothills Hospital

Calgary, Alberta, Canada

Calgary, population 600,000, planned a citywide disaster exercise "Green Cloud" involving a chemical exposure to chlorine, as a test for emergency services (fire, police, ambulance) and hospitals. Five of the six acute care hospitals participated.

The poison center (PC) sought participation in this exercise in order to: 1) implement a recently developed internal disaster plan; and 2) identify hospital and prehospital requirements for PC expertise and resources. The internal disaster plan consisted of a detailed communication and procedure protocol as well as a multi-casualty incident form developed specifically to improve the capture of patient data under disaster circumstances. Miscommunication at the scene during the disaster exercise, with hospitals not being informed specifically of a chemical exposure, resulted in under-utilization of the PC as a source of information/advice. The experience has shown that although hospitals and municipal disaster committees recognize the value of $\mathrm{PC}$ involvement in a chemical disaster, neither group initially considered including the PC in a formal city disaster plan. As a result of this exercise, the $\mathrm{PC}$ has been included in hospital disaster plans and discussions are underway to formalize involvement at a municipal level.

Conclusion: A poison center should seek active involvement with hospital and municipal disaster committees, including participation in disaster exercises, and become an integral part of the EMS response to hazardous materials accidents. It also offers the opportunity to implement internal poison center disaster plans and make appropriate modifications.

\section{1}

\section{Argentine Chemical Information} Center for Emergencies

Gotelli $C A, *$ Grande J, ** Gotelli $D N^{*}$

* Chemical Information Center for Emergencies (CIQUIME)

** Ministero de Salud y Accion Social

Buenos Aires, Argentina

The Chemical Information Center for Emergencies (CIQUIME) is an example of cooperation between the Health Ministry and a private institute. Its main goal is to provide the population with practical and updated information about how to manage chemical emergencies. Other activities include:

1) national hazards mapping;

2) dangerous areas identification;

3) specialized human facilities census;

4) sanitary facilities census;

5) courses and training programs for firefighters and rescue teams; and
6) development of first-aid guides for hazardous

materials incidents.

This paper also includes statistics about incidents, a description of an incident and the measures that were taken, how information is classified, and a brief report about the Argentine situation relative to transportation of hazardous materials.

\section{2}

\section{The Emergency Response Plan of the Japan Poison Information Center in the Event of Chemical Disaster \\ Goto $K$, Ukai $T$, ** Kusakawa $S$, Yamamoto $Y$, ** Yoshioka $T^{+}$ \\ * Japan Poison Information Center \\ Tokyo, Japan \\ ** Osaka Prefectural Senri Critical Care Medical Center \\ *** Nippon Medical School \\ + Osaka University \\ Osaka, Japan}

With the increasing use of hazardous materials in industry, the risk of human-made disasters by those chemical substances has increased. In 1990, more than 300 accidental explosions and spills of hazardous materials occurred in petrochemical complexes and industrial areas in Japan.

As a test case, the Japan Poison Information Center (JPIC) investigated chemicals in Nagoya, one of the petrochemical complexes in Japan, and the need for chemical information by medical facilities in the event of chemical disaster. The main problem was that very many chemicals were handled in that region, but few people were aware of JPIC's activities. Very few medical facilities wanted to obtain information to prepare for disaster prior to being overwhelmed by panicked citizens.

Currently, investigations are being conducted in all coastal regions in Japan and medical associations in affected areas. The present study is aimed at surveying the types of chemicals handled in a particular operation center, the preparation of local government bodies to deal with chemical disasters, and ways in which JPIC can be of service to the region.

\section{3}

\section{Computerized Management of Industrial Incidents}

Magalini SI, Barelli A, Sandroni C

Catholic University School of Medicine

Rome, Italy

When responding to an industrial accident, the initial task is the identification of the hazardous products involved. Numerous publications are available to accomplish it, but only a few of these are computer-based. The Poison Control Center of Catholic University in Rome, in cooperation with the European Centre for Disaster Medicine, currently is building a new database specifically oriented to the identification of all hazardous materials potentially involved in major chemical acci- 